\title{
Modeling situations in an intelligent connected furniture environment
}

\author{
Cedric DEFFO SIKOUNMO, Eric BENOIT, and Stephane PERRIN \\ Université Savoie Mont Blanc, Laboratoire d'Informatique, Systèmes, Traitement de \\ l'Information et de la Connaissance (LISTIC),74944 Annecy-Le-Vieux, France \\ \{cedric.deffo-sikounmo, eric.benoit, stephane.perrin\}@univ-smb.fr
}

\begin{abstract}
The Internet of Thing allows objects and services to interact with each other. The goal of this study is to recognize high level states of the rooms and more generally of the home. We want to be able to obtain intermediate states like "someone is in the kitchen" or "night mode". In the specific case of home activity and state measurement, we consider that a set of furniture units is especially suitable for providing low level information. Recognizing and identifying house states or other high level information can be done using several methods. In this paper, we present an ontology based method. In the following, a situation is considered to be realized when the hypothesis which represents it are fulfilled. Using this approach, we show that multiple instances of situation context are distinguishable.
\end{abstract}

Keywords: Indentification of situation, Ontology, Internet of things, Context, Furniture

\section{Introduction}

The Internet of Thing is perceived as a pervasive presence around us of a variety of things or objects interacting between each other. The given things are physical objects or virtual ones. The physical objects are mainly connected objects. Virtual objects have no existence in the concrete world but they are also able to propose services. The goal of our study is to recognize high level states of the rooms and more generally of the home i.e. normal state, abnormal state or alarm. In addition, we want to be able to obtain intermediate states like "someone is in the kitchen" or "night mode". In the specific case of home activity and state measurement, we consider that furniture units are especially suitable to provide low level information. This is particularly due to their presence around the house and because they are non-intrusive nature. Recognizing and identifying house states or other high level information can be done using several methods. In this paper, we propose to use an ontology-based approach to represent a context made of an aggregation of realized situations. The used ontologies can be supplied and updated directly by sensors. As suggested by [4], we apply inference rules on the graph that represents the concept ontology i.e. the ontology of physical entities. This thus provides high level information. However, inference rules 
are applied on the graph representing the ontology of realized situations. The latter allows the designer to take into consideration dynamic modification of the measured scene without adding new rules. However, if a situation has multiple instances, the problem turns out to distinguish each instance of the others. In this paper, we propose to attach rules on the situation. Using this approach, we show that multiple instances of context within a situation are distinguishable. In the next section on this paper, we discuss about several approaches to model and to represent information of context. In the section 3, we present the ontology based representation of a furniture community. Section 4 presents the representation of situations in a furniture community. Section 5 explains the recognition process, illustrated with an example.

\section{State of the art}

In the literature, several studies were realized on the various situations that can be observed in an intelligent environment. Several studies do not consider the distinction between a situation and a context [3] [16] [17]. The information of context is generally defined as any information being used to characterize the situation of an entity. Such entity can be a person, a place or an object that is considered as relevant for the interaction between a user and an application, including the user and the application themselves [12]. In this paper, which relates to a community of intelligent connected furniture units, we distinguish three types of context information as proposed in [3] :

Raw context: Context whose related information is directly acquired through sensors. For example, the localization, the current time or any environmental parameters.

Interpreted context: Information of context derived from the raw contexts. For example: Sat on a chair; day periods like the morning, the afternoon or the the evening; activities such as sleeping, working.

Live context: Information obtained by recurring combination of the raw contexts and the interpreted contexts which makes sense for a human. For example : working at the office, looking at the TV within the living room.

Several approaches propose to model context information. Tao Gu et al. [16] classified these approaches into three categories:

Application oriented approach: This approach produces models of context for specific applications. These models are generally proprietary models and don't have a formal basis. They don't support the knowledge sharing between the various systems [21].

Model oriented approach: This category of models usually uses conceptual modeling approaches to represent the context. we can quote studies in [18] and [24] which uses the entity-relation model, studies in [19] which uses at the same time the entity-relation model and UML diagrams. Finally, studies in [20] reformulate this model with the extension of "the object-Role Modeling" 
(ORM) [20]. Although this approach takes into account the temporal aspect of context information, it badly supports the generalization of knowledge and the reasoning. In [13], Avelino J. Gonzalez et al offers an intelligent, contextbased behavior representation from the different perceptions of a submarine for training simulations. They use a hierarchical representation of contexts and they add rules to determine the best tactics to adopt depending on the situations that arise.

Ontology oriented approach: The context can be regarded as a kind of specific knowledge. It can be modeled like an ontology. Indeed, the use of ontologies allows not only the context modeling, but also the inference engine based reasoning on the collected data [17].

We will use this latter approach in the following section in order to represent different information from contexts in a community of connected and intelligent furniture units.

\section{Representation of a furnitures community}

\subsection{Presentation}

A community of furniture units is a set of furniture pieces of various species like table, chair, bed, or mirror. These furniture units lead to a given environment. An environment must be understood as any place within where furniture pieces may exist. It can be for example a house, the parts of a house or an office. In this paper, we define a community of intelligent connected furniture units as a set of furniture units, objects and humans interconnected within an environment. In this community, the furniture pieces and the other objects can be intelligent ones i.e. be able to acquire, to handle and to exchange knowledge. In such environment, the furniture pieces, the objects and the humans are considered as the actors of the system. In the case of a house, several furniture units communities related to the different rooms can be identified. A house is generally divided into multiple rooms: living room, room, kitchen, shower, balcony, garden, hollow, garage, etc. A furniture unit can be dedicated to a given room or not, for example a bed or a chair. In the same way, a category of furniture unit can have several variations which are specific to a given room. Moreover, the ontology also includes objects that are not furniture units but that interact with them. For example, a TV interacts with a TV cabinet, so it must be considered into the ontology. In addition, it must be putted forward that human activities related to furniture are part of the most raised activities in a house. As the data acquisition requires sensors, the intelligent connected furniture units found naturally their place in the smart home. The chosen tool for the classification of the data given by sensors is an ontology based one. Such kind of tool already shows its popularity in the field of the engineering of knowledge due to its portability, its evolutionary and its flexibility [16]. 


\subsection{Representation}

Let us first describe the list of concepts and relations used to build the ontology. The figure 1 presents an ontology of a community of smart connected furniture

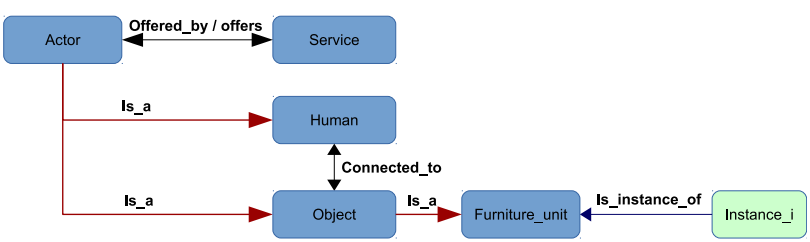

Fig. 1. Ontology of a community of Furniture

pieces. We can raise:

\section{Concepts}

- Actor is the basic concept used to represent the objects, the furniture units and the living beings in a community of connected entities.

- Service is concept representing the various features or capabilities which an actor can offer.

- Human is the concept that represents the human beings in the community.

- Object represents the non-human actors.

- Furniture unit represents a piece of furniture. A furniture piece is described by its style, its category, its functions and by the whole of the parts which compose it. The style is a set of aesthetic criteria and of materials, which helps to recognize the furniture period and its design. The category of a furniture unit refers to its species: tables, chairs, beds, mirrors. A function of a furniture unit leads to the various services it can offer to its user. The construction of a furniture requires various parts which can depend on its category, such as a mounting in the case of a chair or a table, a plate in the case of a table, a back and a seat in the case of a chair. As a result, the ontology of a furniture unit includes the following properties: "Style", "category", "features" and "parts".

- Instance_i represents the individuals i.e. the entities resulting from the instantiation of the various concepts. Except for services, each instance leads to a physical entity.

\section{Relations}

- Is_a: concept $\longrightarrow$ concept is the subsomption relation (hierarchisation). It is a transitive relation.

- Type: individual $\longrightarrow$ concept binds concrete entities to an abstract concept. 
- Part_of: Actor $\longrightarrow$ Actor and Has_part: Actor $\longrightarrow$ Actor are the part-to-whole mereological relations between Actor concepts.

- Offered_by: Service $\longrightarrow$ Actor and offers: Actor $\longrightarrow$ Service are relations establishing a link between an actor and the various services it offers.

- Connected to: Actor $\longrightarrow$ Actor is a topological relation used to formalize the interaction between actors.

- Part of: Actor individual $\longrightarrow$ Actor individual and Has part: Actor individual $\longrightarrow$ Actor individual are the part-to-whole mereological relations between Actor instances. They are inferred from the Is_instance_of and the mereological relations between Actor concepts.

- Connected to: Actor individual $\longrightarrow$ Actor individual is a topological relation use $\bar{d}$ to translate the interaction between actor individuals. The instances of this relation are given by the observation of the individuals, i.e. the physical entities.

This ontology aggregates the various concepts of an environment made of a community of intelligent connected furniture units, connected objects and beings humans. Into the following section, we introduce the representation of the various situations which rise from the interpretation of the contexts of the concept instances.

\section{Representation of the situations in furniture community}

\subsection{Presentation}

We define a situation as follow. A situation is define on a set of actors. Each actor produces sensor data, the raw context, and interprets them to produce its interpreted context. The situation aggregates the raw contexts and the interpreted contexts issued from these actors. Its then appear as an ontology. We distinguish the definition and the realization of situation: The definition is a concept ontology, and the realization is an instance ontology. Actually, the definition of a situation is also called a reference situation and leads to the concept of infon as proposed by Keith D. in [11].

In this section we provide a representation of the various situations that can be found in an environment holding a community of connected and intelligent furniture units. Given an environment we seek to represent the various actors and the relations between them as described in section 3. After this classification stage, the resulting ontology is instantiated and initialized according to the facts of the actor instances. The facts of a furniture unit instance comes from the measured values acquired by its sensors. From this ontology instantiated, we try to recognize the various situations of the environment and the relations which exist between them. Several definitions were proposed in the literature.

In [26], Ye et al. define a situation as an external semantic interpretation of the data sensor. 
- The interpretation term explains the fact that the situations allot significances to the measured values.

- The external term reflects the fact that interpretation is the applications point of view rather than the sensors one.

- The term semantic reflects the fact that an interpretation gives a significance to a set of relations between objects. The concerned sets are themselves defined by data given by sensors.

They alternatively define a situation by the collection of the relevant contexts, by discovering the significant correlations between them, and by the assignment of labels with a descriptive name.

An ontology in the artificial intelligence domain can be defined as the "explicit specifications of a conceptualization"; who is a means of representation, of knowledge sharing and of knowledge reasoning [14]. Several papers gives an outline of the approaches of reasoning on ontology based contexts [4], [8] [26]. As example we can quote SOUPA [6] (Standard Ontology for Ubiquitous and Pervasive Applications) which is based on Cobra-HAVE (Ontology for Context Broker Structures). SOUPA defines a vocabulary to describe the situational conditions such as: "in meeting" or "out of town". Dapoigny and Barlatier [9] formalize the context by using calculations of inductive constructions for the lower layer and ontologies for the upper layer. CONON (CONtext ONtology) is developed by Wang et al. [23]. CONON bases on the concept "context entities", which is a general term from which rises the concepts like "hiring", "person", or "activity". Here, the situations are defined in term of rules.

Example 1. (?u locatedIn Bedroom) \& (Bedroom lightLevel LOW) \& (Bedroom drapeStatus CLOSED) $\longrightarrow$ (?u situation SLEEPING)

Gu et Al. [15] develop the SOCAM system (Service Oriented Context Aware Middelware) where the concept of situation is similar to the CONON one. Anagnostopoulos et al. [2] proposes a "technical situation awareness" based on the combination of ontology and fuzzy logic where the situations are concepts and relations.

Example 2. $\bigwedge_{i=1}^{N} \operatorname{context}\left(x_{i}\right.$,user $) \longrightarrow$ IsInvolvedIn(situation,user), $\mathrm{N}>1$

The example 1 is similar to the example 2 in both cases a situation is represented by rules. Andrey Boytsov et al. [5] bases work which precedes to define a generator of situations. Moreover, they present two concepts of situations: a potential situation is an entity; A potential situation is a relation. Gu and $\mathrm{Al}$. [15] develop system SOCAM (Service Oriented Context Aware Middelware) or the concept of situation is similar to that of CONON.

\subsection{Representation}

Concepts In the proposed approach, we distinguish the definition of a situation from its realization. The realization of a situation is materialized by the existence 
of a sub-graph on individuals linked by the relations Has_part, Part_of and Connected_to. A situation is then defined by a set of rules on concepts.

Thus, the mathematical representation of a situation can be written as follows: let $N$, the set of the nodes, $V$ the set of the relations and $R$ the set of the rules then the situation $S$ can be defined as: $S=G(N, V, R)$.

Example 3. - Sitting situation is the graph where:

$$
\begin{aligned}
& N=\{\text { Chair,Seat,Human }\} \\
& V=\{\text { Part_of,Has_part,Is_instance_of,connected_to }\} \\
& \text { and } \\
& R=\left[r_{1}=\left\{\left(\text { Part_of }(\text { Seat }, \text { Chair }) \vee H a s \_p a r t(\text { Chair,Seat })\right) \wedge \text { Connected_to }(\text { Human, Seat })\right\}\right] .
\end{aligned}
$$

Let Sitting_situation denoting the result of this inference. It represents the fact that the Sitting situation is realized.

A graph of the situation "position seat" is given in figure 2.One can note the various steps of the realization of a situation which will be detailed in the section 5.2. First of all, the step (a) in the figure 2 represents the graph of the situation which refers to sitting. The step (b) shows us a instantiation of this graph with different instances from the concepts "Seat", "Chair" and "Human". The step (c) of the figure 2 established the relations between the instances while basing itself on the observation; an example we can quote the study of the data sensors.
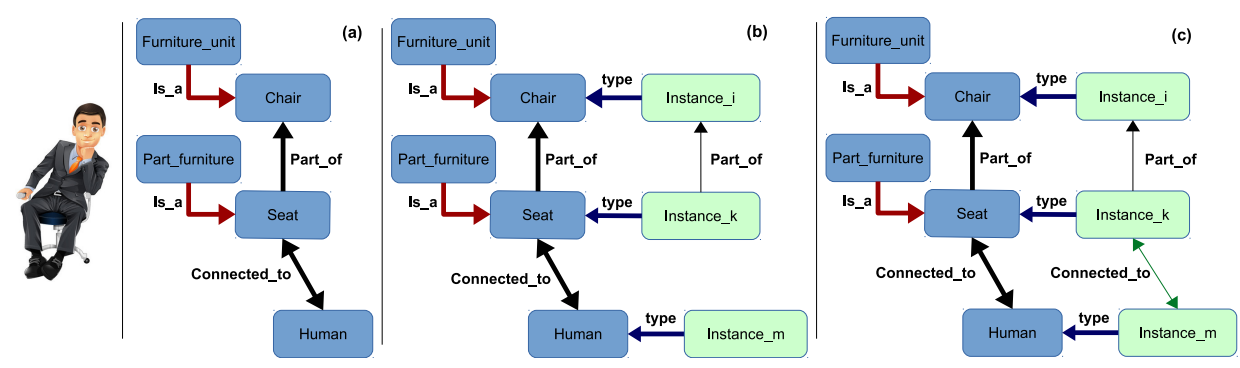

Fig. 2. Sitting situation: (a) graph of the reference situation (b) instantiation of two individuals of types Chair and Human (c) observation of the connected_to relation between individuals

- Working situation is a graph where:

$N=\{$ Sitting_situation, Computer, Human, Table, Support $\}$, $V=\left\{\right.$ Part_of $, H a s \_p a r t, I s \_$instance_of, connected_to, Close_to $\}$ and

$R=\left[r_{1}=\{\right.$ Sitting_situation $\wedge$ Connected_to $($ Computer, Support $) \wedge$ Connected_to(Human,Computer $) \wedge$ Close_to(Chair,Table $)\}$, $r_{2}=\{$ Connected_to(Computer, Support $) \wedge \bar{C}$ onnected_to(Human, Computer $\left.\left.)\right\}\right]$. 
A graph representation of the situation "work" is given by the figure 3 and the relation "Close_to" is describing in [10].

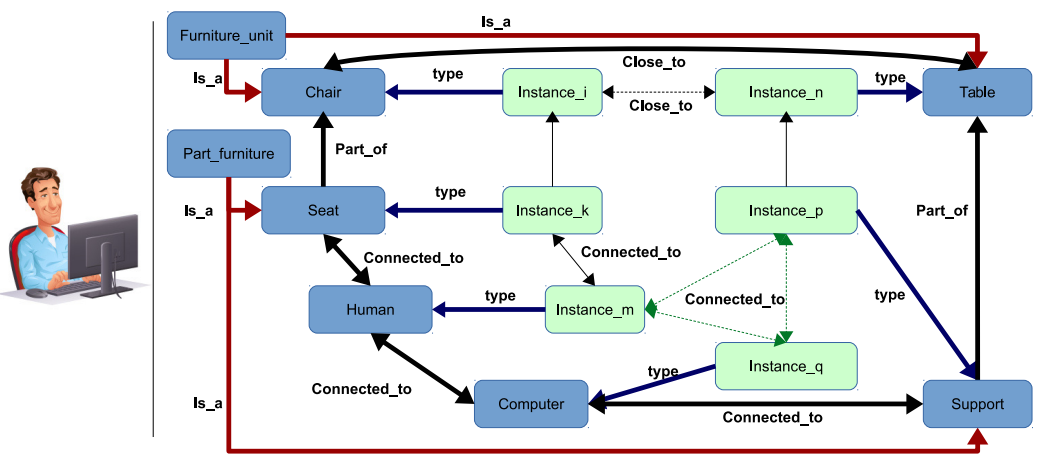

Fig. 3. Graph of the reference situation labeled "work"

\section{Relations}

- The generalization: a situation generalizes another situation, if the realization of the second one implies the realization of the first one. For example the situation "to listen to the music" is a specialization of the situation "entertainment" [25].

- The composition: a situation can result from the composition of other smaller situations; for example the situation "barbecue" breaks up into these two situations "uses a barbecue with gas" and "uses food". In [22] McCowan et al., define a situation of group (for example 'discussion' or 'presentation') like a composition of situations of individual users (for example 'to speak', 'to write').

- of dependence: a situation depends on another situation, if an occurrence of first is determined by an occurrence of the second. The dependence can be one limited time or unlimited, as proposed by [7]. Sometimes, in the long run the dependence can be more useful while deducing from the high level situations. For example, a situation "of going to work" to perhaps better infer a situation "to return to the house of work" than of other situations dependent on short ranges.

- temporal sequence a situation can occur before or after another situation, or interlace with another situation; for example, the situation "taken of the pill" must be realized after the situation "dinner" [1]. 


\section{Situations discovery in furniture units community}

Several techniques for the situation discovery were already proposed. Juan et al. [26] class them in two categories which are:

Specifications based technics These approaches generally build a model of situation with an a priori expert knowledge, and reasons on it with measured values as inputs. As example of technique we can quote Space "Logic programming" "spacial and temporal logic", "ontology" and "Fuzzy logic"

Training based techniques The large variability of the specifications and identifications of the situations, that come from factors like time or the place or the individual,returns inconvenient the specifications based techniques. Training techniques were largely applied to the training of complex associations between the situations and the measured values. In [26] Juan et al. characterizes this training based techniques.

Our contribution in this area consists of a complete situation discovery process based on specifications techniques. In addition, it provides all situation instances in our instantiated concept ontology.

In the literature, the studies on the situation recognition in an pervasive computing environment assume that several basic situations are known without worrying about the way they are obtained. Moreover, a separation between the concept ontology of the sensors and the situations ontology is noticed. In this paper, the representation we propose makes it possible to describe furniture units and the various entities found in their environment. Thus, starting from an instantiation ontology presented in section 3 , the situation references as described in section 4 is used for the situation discovery process. This last process provides the desired realized situations. The complete process is detailed bellow. In this paper, the instance of a concept ontology is an ontology where each constitutive entity is associated with a set of facts. Each fact is a measured value of an entity property.

\subsection{Situation recognition}

The situation recognition technique proposed here assumes the existence of an instance of concept ontology and a set of reference situations. The recognition of situations is first processed from the entities of the instance of our concept ontology and then performed from the situations deduced from this first stage.

Recognition of situations starting from the context instances

Recognition of situations starting from the raw and interpreted context instances is the first way to recognize the various situations of an environment. Knowing a instantiated ontology, the contexts of the various instances are collected. The interpreted contexts also called basic situations are deduced from this collected information.

Example 4. - We want to know if a person sat on a chair. For that, the chair is instrumented with sensors located on his base and back. A person is sitting 
on a chair if he is in contact with the chair on its seat points. Moreover, there are several types of sitting attitudes which depend of the contact between the human and the back of the chair. The figure 2 gives a representation of a basic situation reference.

Recognition of situations starting from the basic situations

High level situations are raised from the basic situations. They are combinations of the basic situations and/or of the entities of our instantiated ontology.

Example 5. - The recognition of a working situation is performs throw the recognition of the basic situations like a person sitting on a chair, a computer on a table, a running computer, a person in contact with the table and the chair near the table. The figure 3 gives a situation reference.

In a general way a situation is known as potentially realizable or recognizable in an instance of our concept ontology $O^{i}$ if for any item of the set of the whole nodes situation $N$ there exists an instance in $O^{i}$.

Proposition 1. $S=G(N, V, R)$ is a potential situation (a situation definition) if: $\forall c \in N, \exists i ; i$ instance_of $c$ and $i \in O^{i}$.

The recognition of situations makes it possible to present the various potential situations of the system, without being certain of their realization.

\subsection{Realization situations}

Given a instance ontology and a set of potential recognized situations, a situation is said to be realized if there exists at least a subgraph of the instance ontology graph that respects at least one of the rules of our reference situation.

Proposition 2. A situation $S=G(N, V, R)$ is realized if: $\exists G_{i}\left(N_{i}, V, r_{i}\right) ; r_{i}$ is verified, with $N_{i}=\{i /(i$ is_instance_of $c) \wedge(c \in N)\}$ and $r_{i} \in R . G_{i}$ is also called instance of the reference situation $S$.

To discover situation in an environment of intelligent connected furniture our system must carry out two stages which are:

Step 1 Recognition of all potential situations based on a list of reference situations.

Step 2 Discovering the situations realized starting from the potential situations extracted in step 1.

Thus, this approach enables us to list all the situations of an intelligent connected furniture environment. Moreover, for each new entity, the same process is reiterated. That makes this approach dynamic in time. 
Input: $O^{i}$ an ontology instance

Input: $L_{R} S$ a list of the reference situations

Output: $L_{P} S$ a list of the potential situations

foreach $s \in L_{R} S$ do

if $\left(\forall c \in N(s), \exists i ; i\right.$ instance_of $c$ and $\left.i \in O^{i}\right)$ then

। $L_{P} S \leftarrow L_{P} S+s$

end

end

return $L_{P} S$

Algorithm 1: Discovered potential situations

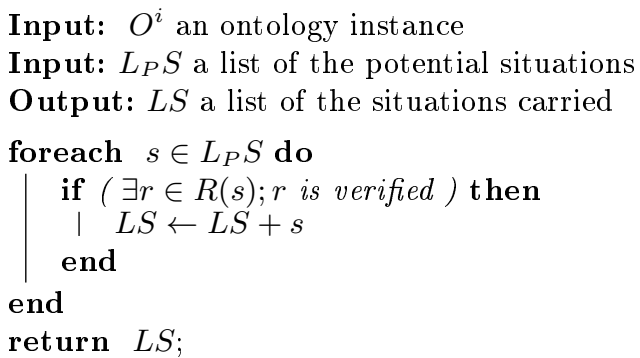

Algorithm 2: Discovered situations realized.

\subsection{Application}

In a first time, let an ontology instance describing an environment made up of three humans and two chairs and a table as described in the figure 4 . In a second time the same instance of concept ontology with new relations as shown in the figure 5 is given. We seek to recognize the various situations in which the actors of our environment are concerned. For that, we define the following situations references:

- Working situation is a graph or: $N=\{$ Sitting_situation, Human,Table $\}$, $V=\{$ Part_of,Is_instance_of, connected_to,Close_to $\}$ and $R=\left[r_{1}=\right.$ $\{$ sitting_situation $\wedge$ Connected_to $($ Human, $\bar{T}$ Table $) \wedge C \overline{l o s e}$ to $($ Chair, Table $)\}, r_{2}=$ $\{$ Connected_to(Human, Table $)\}]$.

- Sitting situation is a graph or:

$N=\{$ Chair, Seat, Human $\}, V=\{$ Part_of,Is_instance_of, connected_to $\}$ and $R=\left[r_{1}=\left\{\left(\right.\right.\right.$ Part_of $($ Seat,Chair $) \vee \overline{H a s} \_$part $($Chair, Seat $\left.)\right) \wedge$ Connected_to $($ Human,Seat $\left.\left.)\right\}\right]$.

- Close to situation is a graph or: $N=\{$ Chair, Table $\}, V=\{$ Close_to $\}$ and $R=\left[r_{1}=\{(\right.$ Close_to(Seat, Chair $\left.)\}\right]$.

By applying the steps presented in section 5.2 we have the following results:

- case of the figure 4

Step 1 (discovery of all potential situations) result of algorithm 1:

\{Close to situation, Sitting situation, Working situation\}

Step 2 (discovered of realized situations) result of algorithm 2:

$\{$ Sitting situation(Human_1, Chair_2), Sitting situation(Human_2, Chair_1)\} 


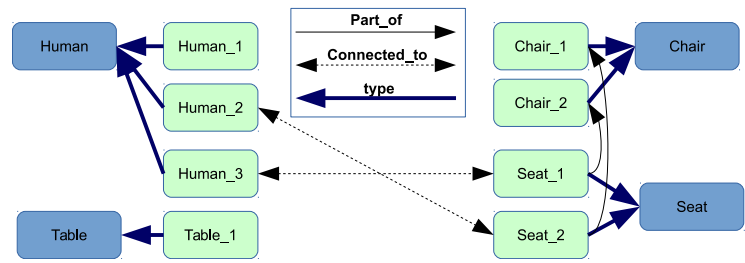

Fig. 4. Example of instance of an ontology representing an environment made up of chairs and humans

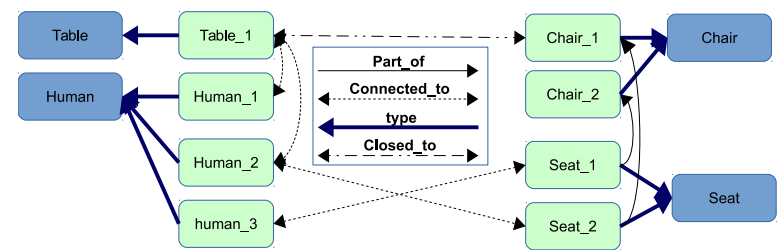

Fig. 5. Example of instance of an ontology representing an environment made up of chairs, the table and humans

- case of the figure 5

Step 1 (discovery of all potential situations) result of algorithm 1:

\{Close to situation, Sitting situation, Working situation\}

Step 2 (discovered of realized situations) result of algorithm 2:

\{Sitting situation(Human_1, Chair_2), Sitting situation (Human_2, Chair_1),

Close to situation(Table_$\overline{1}$, Chair_ 1 ), working situation(Human_$\left.\left.{ }_{-}\right)\right\}$.

\section{Conclusion and perspectives}

In this paper we propose a generic representation of the situations of a community of intelligent connected furniture units. For that, we chose a representation of the physical entities by using ontologies. This representation enables us to emphasize the various parts of a furniture and another entities which can collaborate or interact with furniture units. From the description of community of furniture units, we seek to recognize the various states of our community. Thus, to recognize such states, reference situations are modeled by graphs made of nodes and relations and by rules. From this representation of situations references, we define algorithms which make it possible to find potentially realizable situations and actually realized situations. Given examples illustrate the use of the proposed algorithms in order to provide desired actually realized situations. The future work for the improvement of the presented study will concern the discovery of new situations in an autonomous way and the association of the masses to the various elements of the graph of a situation. This last point of 
improvement will make possible to perform uncertainty based reasoning using theories like: the probability theory, the fuzzy sub-set theory or the evidence theory.

\section{$7 \quad$ Acknowledgments}

This research is supported by the Universite Savoie Mont Blanc, the Association des Pays de Savoie and Miliboo Corporation.

\section{References}

[1] Workshops Proceedings of the 7th IEEE International Conference on Data Mining (ICDM 2007), October 28-31, 2007, Omaha, Nebraska, USA. IEEE Computer Society (2007)

[2] Anagnostopoulos, C., Ntarladimas, Y., Hadjiefthymiades, S.: Situational computing: An innovative architecture with imprecise reasoning. Journal of Systems and Software 80(12), 1993-2014 (2007)

[3] Attard, J., Scerri, S., Rivera, I., Handschuh, S.: Ontology-based situation recognition for context-aware systems. In: I-SEMANTICS 2013 - 9th International Conference on Semantic Systems, ISEM '13, Graz, Austria, September 4-6, 2013. pp. 113-120 (2013)

[4] Bettini, C., Brdiczka, O., Henricksen, K., Indulska, J., Nicklas, D., Ranganathan, A., Riboni, D.: A survey of context modelling and reasoning techniques. Pervasive and Mobile Computing 6(2), 161-180 (2010)

[5] Boytsov, A., Zaslavsky, A.B., Eryilmaz, E., Albayrak, S.: Situation awareness meets ontologies: A context spaces case study. In: Modeling and Using Context - 9th International and Interdisciplinary Conference, CONTEXT 2015, Lanarca, Cyprus, November 2-6, 2015. Proceedings. pp. 3-17 (2015)

[6] Chen, H., Perich, F., Finin, T.W., Joshi, A.: SOUPA: standard ontology for ubiquitous and pervasive applications. In: 1st Annual International Conference on Mobile and Ubiquitous Systems (MobiQuitous 2004), Networking and Services, 22-25 August 2004, Cambridge, MA, USA. pp. 258-267 (2004)

[7] Choudhury, T., Quigley, A.J., Strang, T., Suginuma, K. (eds.): Location and Context Awareness, 4th International Symposium, LoCA 2009, Tokyo, Japan, May 7-8, 2009, Proceedings, Lecture Notes in Computer Science, vol. 5561. Springer (2009)

[8] Compton, M., Henson, C.A., Neuhaus, H., Lefort, L., Sheth, A.P.: A survey of the semantic specification of sensors. In: Proceedings of the 2nd International Workshop on Semantic Sensor Networks ( SSN09), collocated with the 8th International Semantic Web Conference ( ISWC-2009), Washington DC, USA, October 26, 2009. pp. 17-32 (2009)

[9] Dapoigny, R., Barlatier, P.: Formalizing context for domain ontologies in coq. In: Context in Computing - A Cross-Disciplinary Approach for Modeling the Real World, pp. 437-454 (2014)

[10] Deffo Sikounmo, C., Benoit, E., Perrin, S.: States measurement in a context of intelligent connected furnitures. In: 2016 Joint IMEKO TC1-TC7-TC13 Symposium "Metrology across the Sciences: Wishful Thinking?". To be published in IoP Science, Berkeley Evaluation and Assessment Research Center, Berkeley, United States (Aug 2016) 
[11] Devlin, K.: Situation theory and situation semantics. In: Logic and the Modalities in the Twentieth Century, pp. 601-664 (2006)

[12] Dey, A.K.: Understanding and using context. Personal and Ubiquitous Computing 5(1), 4-7 (2001)

[13] Gonzalez, A.J., Ahlers, R.: Context-based representation of intelligent behavior in training simulations. Transactions of the Society for Computer Simulation 15(4), 153-166 (1998)

[14] Gruber, T.: Ontology. In: Encyclopedia of Database Systems, pp. 1963-1965 (2009)

[15] Gu, T., Pung, H.K., Zhang, D.: A service-oriented middleware for building context-aware services. J. Network and Computer Applications 28(1), 1-18 (2005)

[16] Gu, T., Wang, X.H., Pung, H.K., Zhang, D.Q.: An ontology-based context model in intelligent environments. In: IN PROCEEDINGS OF COMMUNICATION NETWORKS AND DISTRIBUTED SYSTEMS MODELING AND SIMULATION CONFERENCE. pp. 270-275 (2004)

[17] Guermah, H., Fissaa, T., Hafiddi, H., Nassar, M., Kriouile, A.: An ontology oriented architecture for context aware services adaptation. CoRR abs/1404.3280 (2014)

[18] Harter, A., Hopper, A., Steggles, P., Ward, A., Webster, P.: The anatomy of a context-aware application. Wireless Networks 8(2-3), 187-197 (2002),

[19] Henricksen, K., Indulska, J., Rakotonirainy, A.: Modeling context information in pervasive computing systems. In: Pervasive Computing, First International Conference, Pervasive 2002, Zürich, Switzerland, August 26-28, 2002, Proceedings. pp. $167-180$ (2002)

[20] Henricksen, K., Indulska, J., Rakotonirainy, A.: Generating context management infrastructure from high-level context models. In: In 4th International Conference on Mobile Data Management (MDM) - Industrial Track. pp. 1-6 (2003)

[21] Kindberg, T., Barton, J.: A web-based nomadic computing system. Comput. Netw. 35(4), 443-456 (Mar 2001)

[22] McCowan, I., Gatica-Perez, D., Bengio, S., Lathoud, G., Barnard, M., Zhang, D.: Automatic analysis of multimodal group actions in meetings. IEEE Trans. Pattern Anal. Mach. Intell. 27(3), 305-317 (2005)

[23] Wang, X., Zhang, D., Gu, T., Pung, H.K.: Ontology based context modeling and reasoning using OWL. In: 2nd IEEE Conference on Pervasive Computing and Communications Workshops (PerCom 2004 Workshops), 14-17 March 2004, Orlando, FL, USA. pp. 18-22 (2004)

[24] Wu, H., Siegel, M., Ablay, S.: Sensor fusion for context understanding. In: Instrumentation and Measurement Technology Conference, 2002. IMTC/2002. Proceedings of the 19th IEEE. vol. 1, pp. 13-17 vol.1 (2002)

[25] Ye, J., Coyle, L., Dobson, S.A., Nixon, P.: Representing and manipulating situation hierarchies using situation lattices. Revue d'Intelligence Artificielle 22(5), 647-667 (2008)

[26] Ye, J., Dobson, S., McKeever, S.: Situation identification techniques in pervasive computing: A review. Pervasive and Mobile Computing 8(1), 36-66 (2012) 